\title{
Sound Absorption Properties Evaluation and Analysis of Recycled Tyre Textile Fibre Waste
}

\author{
Robert RUŽICKIJ ${ }^{*}$, Tomas ASTRAUSKAS ${ }^{2}$, Sarma VALTERE $^{3}$, \\ Raimondas GRUBLIAUSKAS ${ }^{4}$ \\ ${ }^{1,2,4}$ Vilnius Gediminas Technical University, Saulètekio avenue 11, Vilnius, Lithuania \\ ${ }^{3}$ Riga Technical University, Kalku iela 1, Riga, Latvia
}

\begin{abstract}
Modern world is exposed to various environmental concerns which are closely related to human health condition. Since the automatization, world became vulnerable to the noise and the waste amounts generated. World Health Organization report states, due to noise, Western Europe each year loses approximately 1 million of healthy life years. However, noise is not the only concern. Tyres, since they were banned from the landfills, became enormous problem in a modern society. Approximately $\mathbf{2 . 6}$ million tonnes of tyres are generated each year in Europe, out of which 320000 tonnes of Recycled Tyre Textile Fibre (RTTF) waste. Practically, rubber granules and metals extracted from tyre can be reused, however reusing RTTF is a challenge. The main focus is on the possibility of reuse of RTTF in buildings for acoustical comfort improvement. The determination of sound absorption is implemented by experimental research, based on ISO 10534 standard, involving five types of sound absorbing materials. It was concluded that RTTF has a great potential in use for sound absorption structures and can be an alternative substitute to non-renewable and non-recyclable materials.
\end{abstract}

Keywords - Fibrous sound absorbing materials; mineral wool; sound absorption coefficient; tyres waste

\section{INTRODUCTION}

Rapid growth and urbanisation of the cities prone inhabitants to be exposed to the noise pollution. Due to the willingness of builders to save money on using less qualitative and cheaper materials, new residents suffer from insufficient noise insulation. Concerning this reason many residents complain on the noise coming from adjoining apartments, audible conversations, and other unwanted and disturbing sounds.

Each year, only in Europe, approximately 2.6 million tonnes of tyres are disposed, out of which about 320000 tonnes of Recycled Tyres Textile Fibre (RTTF) waste is generated. Rubber has been successfully reused for airborne sound insulation in buildings [1], roads surface improvement [2], while metals reused in production, and constructions.

Although, to reduce noise levels at the living environment is complicated, solutions could be applied. Mineral wools, expanded polystyrene, rubber and plaster tiles the most common solutions for building acoustics [3], however mostly produced from primary raw materials. Tyre textile fibre waste, and in general textile waste, has a potential for use in sound

* Corresponding author.

E-mail address: robert.ruzickij@stud.vgtu.lt

(C)2020 Robert Ružickij, Tomas Astrauskas, Sarma Valtere, Raimondas Grubliauskas.

This is an open access article licensed under the Creative Commons Attribution License (http://creativecommons.org/

licenses/by/4.0), in the manner agreed with Sciendo. 
insulation [4]. According to the [4] by obtaining different composites, it was concluded that textile fibre wastes improves sound absorption of the material.

Long and excessive sound level at the living environment causes health deterioration, which can lead to the fatal consequences. Continuous excessive noise sets off the body's acute stress response, which raises blood pressure and heart rate, potentially mobilizing a state of hyperarousal [5]. The response to that can lead to cardiovascular disease and other health issues: sleep disturbance [6], annoyance, tinnitus or even hearing impairment and hearing loss [7].

End-of-life Tyres are one of the main sources of waste in End-of-life Vehicles. Alone in Europe, each year about 2.6 million tonnes are disposed, from which about $10 \%$ of total weight amount comprises Recycled Tyre Textile Fibre, which is about 320000 tonnes [8].

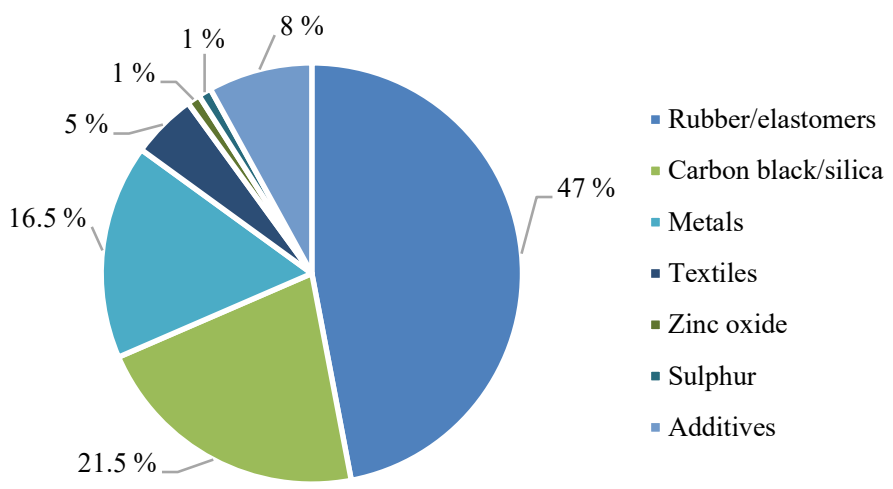

Fig. 1. Composition by Mass of EU Car [4] (Note: the amount of textile fibre in Cars varies $5-10 \%$ (mostly accepted as $10 \%)$.

It is important to understand the composition of the End-of-life Tyre. Authors [9] and [10] in their researches present the composition and amount of material of total tyre weight. Fig. 1 presents the composition and materials part of total amount of tyre.

According to the Fig. 1 detailed composition by mass of car tyres in the EU Market. The data reflects the generic (not patented) formula for tyres produced for European Market. Material composition varies by category, and nominally in which continent it is produced, although ratio of materials stays relatively very similar [10].

The nylon could be considered as the thermoplastic [11]. Authors [12] have noted that plastics these days is a large-scale problem, but especially - microplastics pollution. Pollution by the microplastics rapid growth sends alerts that solutions must be acted now. [13] in their research found out that plastics accumulation in the environment is high. [14] in their research presented information that in the world ocean, in the depths of more than 2000 meters, the microplastics were found. The wildlife of the oceans are ingesting thermoplastics, viscose and other kinds of plastics [14].

\subsection{Sound Absorption in Fibrous Materials}

Sound absorption is a process during which energy of sound is reduced, when the sound wave passes through the medium and loses energy there (Fig. 2). The main characteristics of 
sound absorption is - sound absorption coefficient $(\alpha)$ - which defined as ratio of absorbed energy in absorbent and incident energy [15].

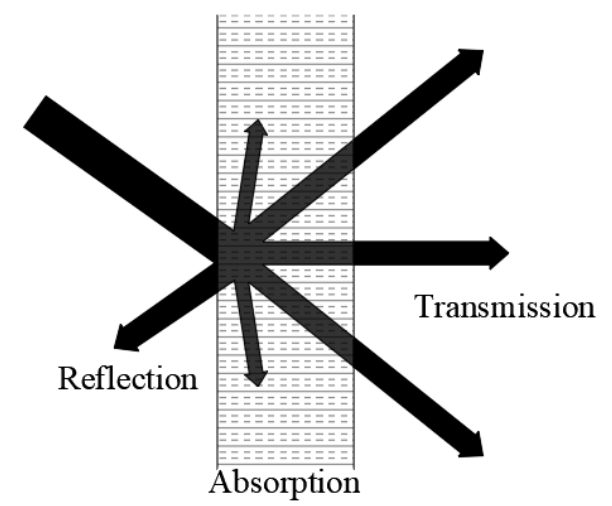

Fig. 2. Propagation of sound wave in fibrous materials [16].

Important to note, that the higher percentage of energy is absorbed into the material, the better absorption is, and less energy emitted back to the environment. The principle of finding of materials' $\alpha$ absorption coefficient is calculated:

$$
\alpha=\frac{E_{A}}{E_{I}},
$$

where $E_{A}$ absorbed wave energy, W, and $E_{I}$ incident wave energy, W [17].

$$
\alpha=1-\frac{E_{R}}{E_{I}},
$$

where $E_{R}$ reflected wave energy, W, and $E_{I}$ incident wave energy, W [17].

$$
\alpha=1-|R|^{2}
$$

where $R$ sound reflection coefficient [17].

$$
\alpha=\frac{I_{A b s}}{I_{I n c}},
$$

where $I_{A b s \text {. }}$ sound intensity absorbed, W/m², $I_{\text {Inc. }}$ incident sound intensity, W/m² [17].

Different sound absorbing materials are classified by its' abilities to absorb sound wave. Material considered as a good sound absorbing material which absorbs and transmits more sound wave energy rather than reflects. Main parameters that influence absorption of the materials, are:

- thickness of the material;

- density of the material;

- porosity of the material [18].

Material thickness. The thickness of the absorbing material has an influence in sound absorption, although it is only significant at low frequencies, which ranges from 50 to 
$2000 \mathrm{~Hz}$, and mostly has no significant impact on high frequency $>2000 \mathrm{~Hz}$. The reason is, that the low frequency sound waves has higher wavelength [19]. The result of thicker absorbing material is better sound absorption in low frequencies. Crucial point is the materials thickness has to be at least $1 / 4$ of the wavelength to provide an effective sound absorber and this is considered to be condition for the lower frequency [17].

Material density. Sound absorption is influenced by the materials density. By the acoustic impedance determined, that reflection of the material depends on the density, since denser it is, the surface becomes more rigid. Widely it is considered that as higher density is, the higher sound absorption, however the phenomenon occurs due to mass increase [20]. At higher frequencies materials ability to absorb sound decreases due to thickness, which is dominant factor [17]. The critical frequencies of 1000 to $4000 \mathrm{~Hz}$ strictly limits absorption of the sound wave when the incident sound wave is in one phase with reflected wave, the coincidence dip phenomenon occurs [21]. The coincidence dip phenomenon can be explained as unison of the frequency at which material vibrates and the frequency of incident sound wave [22].

Material which has high density, has a larger surface per volume unit and fibres content [23]. As the fibres content increases, surface of the absorbent material is exposed to more energy loss due to friction, and sound waves are converted to heat energy [24]. It was noted by the authors [17] that with decreased size of fibres, airflow increases in-between and friction increases too. The statement is that finer particles are better on sound absorption, rather than coarser.

Material porosity. Porosity is the factor, which is simply called volume of the pores in the total volume of material. The role of pores is important, whereas acts crucial part of dispersion of medium sound waves. The principle of behaviour of sound wave reaching a pore is that when it comes into contact with the pore it meets air molecules, which starts to vibrate. The wave loses its' energy due to interaction to the air molecules in the pores and the mechanical energy is transformed to heat due to thermal and viscous losses inside the pores' channels [25].

The parameter of pore, which makes absorption even better is that pores containing continuous channels are tend to better sound absorbing due to multiple interaction with the wall of the pore [17]. The other parameter of the pore - diameter - the smaller it is, the better absorption, due to at high frequencies of sound wave large gaps has no profound effect on absorption [26].

The studies were conducted on use of RTTF in concrete [27], asphalt production [28], and polypropylene products [29] on mechanical properties improvement, however acoustic properties of RTTF are not tested yet. The reuse of RTTF is proposed within this paper. The aim of this paper is to characterise and compare RTTF waste sound absorption with wellknown mineral wools in the field. Experimental research was performed using standard transfer function method with two microphone impedance tube system. The paper is organized as follows: in Sec. 2 research methodology is provided, in Sec. 3 main results and discussion, and in Sec. 4 conclusions of the study are presented.

\section{Methodology Of THE RESEARCH}

For the experimental part three different materials were used: rock wool (RW), glass wool (GW) (conventional) and RTTF (unconventional). RTTF used for the experiment was brought from the tyre recycling factory from Gargždai town, Lithuania. During the recycling process, tyre parts are separated and RTTF is being collected into the bags. The material was dried at $80{ }^{\circ} \mathrm{C}$ temperature in a drier, to exclude moisture accumulated during the storage time. Drying 
process took in total 5 days.

After the drying process was completed, raw RTTF was mechanically treated into three fractions. Treatment was selected due to raw primary material is received in large pieces, which can be seen in Fig. 3a. Afterwards, by shredding process, raw primary material was comminuted into smaller particles, since raw material was received as packed large pieces (Fig. 3b). The rubber particles remained, which are seen in Fig. 4b. Second treatment method was purification (Fig. 3c). Primary raw material was purified from rubber particles remain by sifting through a sieve. In raw RTTF rubber particles remains make up to $10 \%$ of total weight. The rubber particles were removed as much possible, leaving the smallest possible amount.

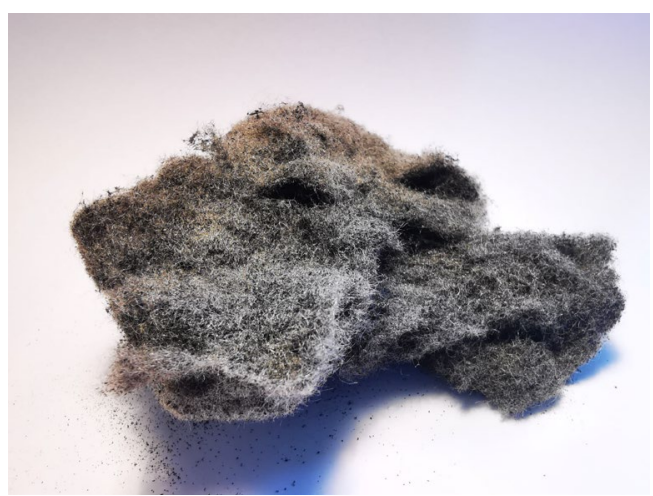

a)

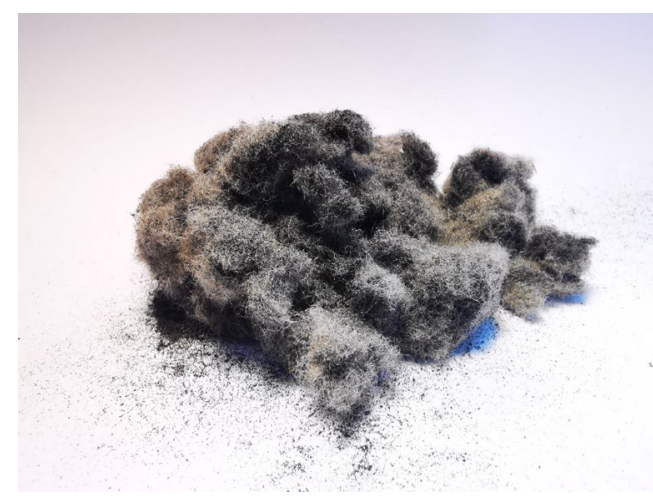

b)

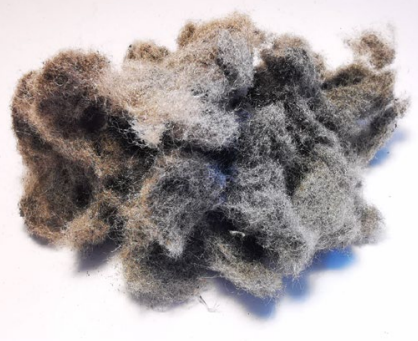

c)

Fig. 3. RTTF samples: a) - raw primary RTTF; b) - shredded RTTF; c) - purified RTTF

The inner area size of the impedance tube shell is $10 \times 10 \mathrm{~cm}$. Since rock and glass wools are pre-shaped in advance at the factory, they only needed to be cut into required size. RTTF was inserted into the tube to contain $4 \mathrm{~cm}$ thickness. Total amount of five samples were investigated: rock wool, glass wool, raw RTTF, shredded RTTF, and purified RTTF.

Sound absorption coefficient $\alpha$ measurement equipment called - impedance tube, is used. Impedance tube (Fig. 4) - an equipment used for investigation of sound absorption and reflection of material, when the sound wave moves towards the sample. Impedance tube made of the thick shell, sound source, microphones, and the space for sample. Using impedance tube, non-acoustic parameters of the material can be determined by indirect methods porosity, tortuosity and airflow resistivity [30]. 


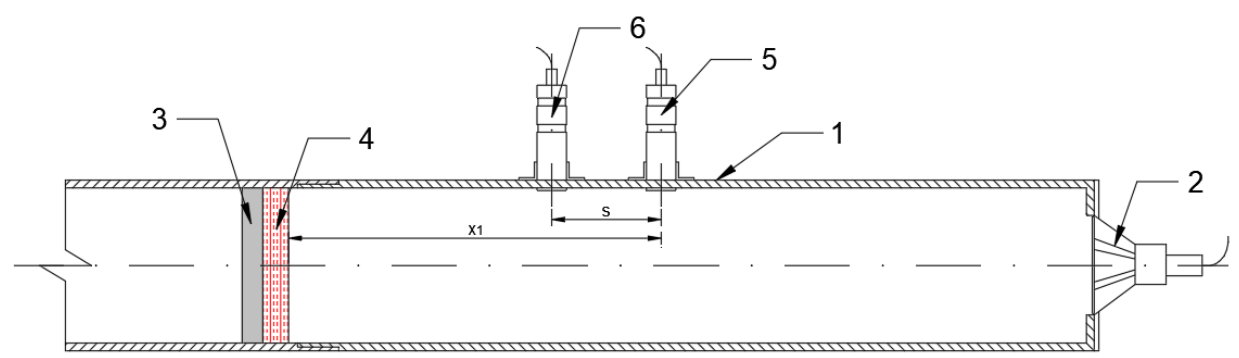

Fig. 4. Impedance tube principle scheme: 1 - impedance tube shell (acrylic glass), 2 - sound speaker, 3 - rigid backing, 4 - sound absorption sample, 5 - microphone No. 1, 6 -microphone No. 2

Impedance tubes differ in their parameters depending on the properties to be investigated. The main parameter - diameter. Regarding the diameter, the frequency range in which sound absorption coefficient can be measured, determined [31].

Method description according to the ISO 10534-1 standard:

1. Ambient air temperature in impedance tube measured and the sound wave speed is determined. The variation of the temperature in impedance tube cannot differ by more than 1 Kelvin, otherwise if a drastic change is found, the results are considered unrepresentative and the experiment must be repeated;

2. The sample is tightly installed before the rigid backing and all connecting parts are sealed with lithium grease, to prevent sound leaking;

3. Using sound speaker in impedance tube, pink sound pressure level is released. The sound level in impedance tube must be $10 \mathrm{~dB}$ higher than the background noise during the experiment;

4. The released sound from the sound source is recorded by the microphones (maximum and minimum sound pressure levels);

5. Sound absorption and reflection coefficients are calculated using software and mathematical-physical formulas;

6. All measurements are repeated 3 times in order to calculate standard deviation and have representative data [32].

After the measurements, the pressure is determined at each frequency by transfer function methods (ISO 10534-2 standard):

$$
H_{12}=\frac{p_{2}(f)}{p_{1}(f)},
$$

where

$H_{12} \quad$ Transfer function between microphones No. 1 and No. 2;

$p_{2} \quad$ Pressure recorded by the second microphone, $\mathrm{Pa}$;

$p_{1} \quad$ Pressure recorded by the first microphone, $\mathrm{Pa}$;

$f \quad$ Frequency, $\mathrm{Hz}$ [33].

$$
k_{0}=\frac{2 \pi f}{c_{0}},
$$


where

$k_{0} \quad$ Wave number in the air;

$f \quad$ Frequency, Hz;

$c_{0} \quad$ Sound wave speed in the air, $\mathrm{m} / \mathrm{s}$ [33].

$$
H_{\mathrm{I}}=e^{-\mathrm{j} k_{0} s}
$$

where $H_{\mathrm{I}}$ is incident wave transfer function, and $s$ is distance between microphones, $\mathrm{m}$ [33].

$$
H_{\mathrm{R}}=e^{j k_{0} s},
$$

where $H_{\mathrm{R}}$ is reflected wave transfer function, and $s$ is distance between microphones, $\mathrm{m}$ [33].

Sound wave reflection is calculated by the Eq. (9):

$$
R=\frac{H_{12}-H_{\mathrm{I}}}{H_{\mathrm{R}}-H_{12}} e^{2 \mathrm{j} k_{0} x_{1}},
$$

where

$R \quad$ Sound reflection coefficient;

$H_{\mathrm{I}} \quad$ Incident wave transfer function;

$H_{\mathrm{R}} \quad$ Reflected wave transfer function;

$k_{0} \quad$ Wave number;

$x_{1} \quad$ Distance between microphone No. 1 and the sample $\left(x_{1}=190 \mathrm{~mm}\right)$ [33].

Sound absorption ability is inverse value of sound reflection coefficient of the materials. Sound absorption for the flat waves is calculated by the Eq. (10):

$$
\alpha=1-|R|^{2},
$$

where $\alpha$ is materials sound absorption coefficient [33].

To calculate transfer function MATLAB script was developed. Directly measured parameters - sound pressure levels in each microphone and phase shift.

In the Sec. 3 the results of the research, based on the methodology, are described.

\section{RESUlts AND DiscuSSION}

Sound absorption measurement results of RW and GW are presented in Fig. 5. According to the results, lowest sound absorption was obtained in the low frequency band, i.e. at $250 \mathrm{~Hz}$ frequency, and sound absorption results were for rock wool -0.24 , and for glass wool 0.25 . The highest measured materials sound absorption coefficient was obtained at $1600 \mathrm{~Hz}$ frequency, with increasing frequency. The glass wool result in the $1600 \mathrm{~Hz}$ frequency was 0.77, while RW showed higher sound absorption coefficient 0.93 .

Sound absorption coefficient increases with the frequency. At $250 \mathrm{~Hz}$, the sound wavelength is approximately 1.38 meters, and comparing to $1600 \mathrm{~Hz}$, where sound wavelength is approximately 0.22 meters. Absorbing materials are effective when materials thickness is $1 / 4$ of wavelength at the selected frequency. 


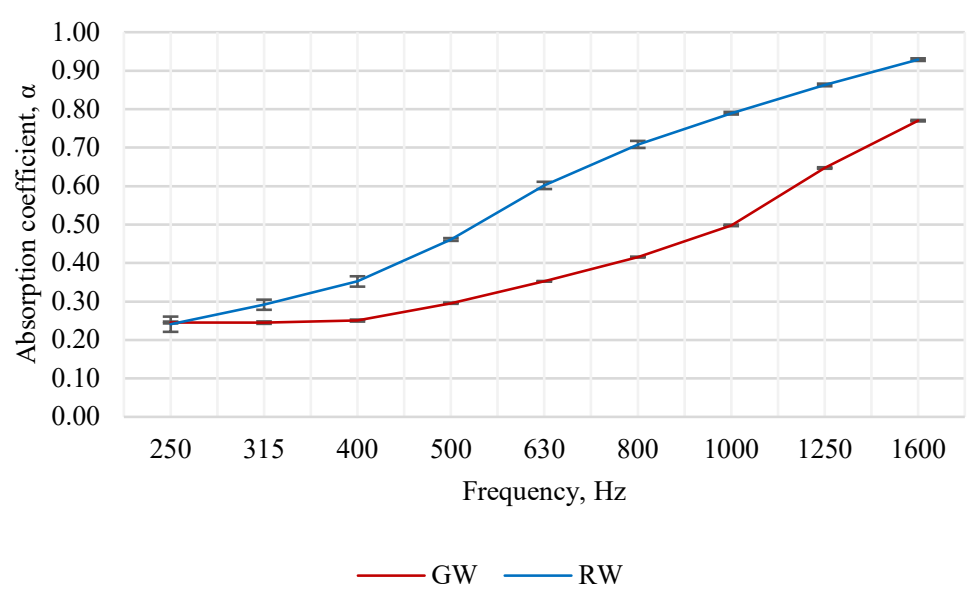

Fig. 5. Comparison of two conventional materials: rock wool vs. glass wool.

The difference of two materials at low frequency, $250 \mathrm{~Hz}$ frequency band, sound absorption is quite similar, by difference of 0.01 . While as the frequency of the sound wave increases, the difference between two materials becomes clearly visible. The difference becomes marginal from $315 \mathrm{~Hz}$, and the biggest difference is observed at $800 \mathrm{~Hz}$ frequency (as well as at $1000 \mathrm{~Hz}$ frequency), where RW absorption coefficient is 0.71 and GW absorption coefficient is 0.42 which makes difference of 0.29 .

Sound absorption coefficient of GW at the highest peak is 0.77 . Such phenomenon occurs due to: materials thickness, density, and porosity. In this case, density plays an important role, since GW density is relatively low, comparing to, for instance RW. Average density of glass wool was $16.0 \mathrm{~kg} / \mathrm{m}^{3}$ and rock wool density was $38.0 \mathrm{~kg} / \mathrm{m}^{3}$. For fibrous materials, density is one of the important parameters defining the sound absorption coefficient, as higher the density is, the higher sound absorption. Higher density of fibrous materials means more compressed fibres which results to higher sound absorption coefficient values.

Results of sound absorption measurements for shredded, raw, and purified RTTF are presented in Fig. 6.

According to the obtained measurements results, the lowest sound absorption coefficient was obtained in all samples at $250 \mathrm{~Hz}$ frequency, $\alpha=0.28$. The maximum sound absorption coefficient was obtained in all samples at $1600 \mathrm{~Hz}$ frequency. Shredded RTTF sound absorption coefficient was 0.95 , raw RTTF sound absorption coefficient was 0.92 , and purified RTTF sound absorption coefficient was 0.97 .

According to the results in the figure, small difference can be obtained between the different mechanically treated samples. Results show that in mid-frequency $250 \mathrm{~Hz}$ to $1000 \mathrm{~Hz}$, raw RTTF showed higher sound absorption coefficient values. Better performance can be explained that raw RTTF was more packed and compressed, which made it denser. While preparing other samples, they were shredded, purified - mechanically treated, which led to material being less dense. 


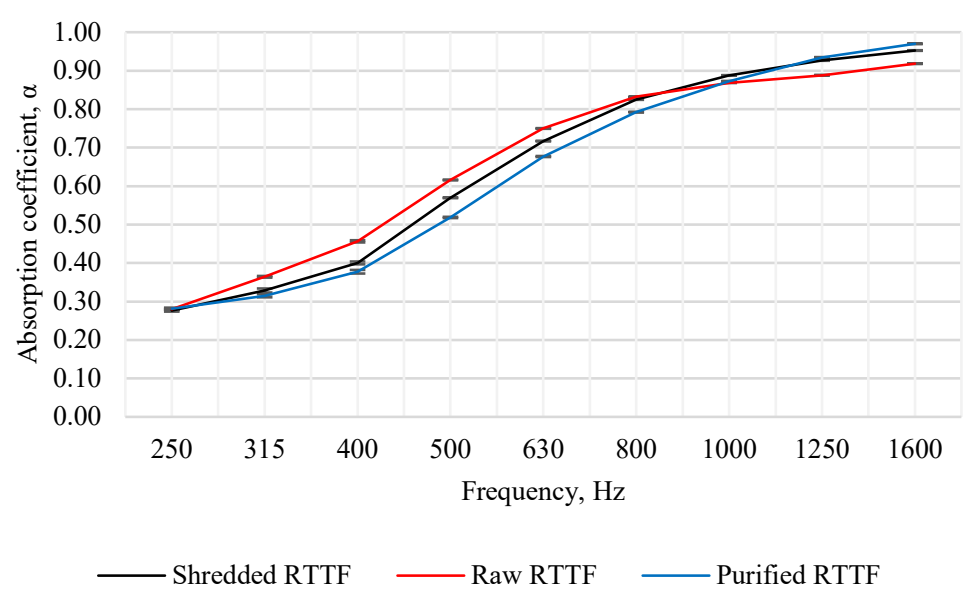

Fig. 6. Comparison of non-conventional materials options: shredded RTTF vs. Raw RTTF vs. purified RTTF.

In measured frequency range, raw RTTF performance on sound absorption is better, comparing to shredded and purified RTTF. At higher frequency from $1000 \mathrm{~Hz}$ sifted RTTF moves forward and its absorption comparing to shredded and raw RTTF is higher. According to the results, at frequency bands $1000-1600 \mathrm{~Hz}$, better performance identified in purified sample of RTTF, however in overall at this frequency range all samples perform very similarly.

Table 1 represents comparison of all measured samples. According to the results in the table the most efficient performance of all samples can be identified.

TABLE 1. COMPARISON OF MEASURED SAMPLES

\begin{tabular}{llllllllll}
\hline & \multicolumn{1}{l}{ Frequency, $\mathbf{H z}$} \\
\cline { 2 - 9 } Sound absorption coefficient & $\mathbf{2 5 0}$ & $\mathbf{3 1 5}$ & $\mathbf{4 0 0}$ & $\mathbf{5 0 0}$ & $\mathbf{6 3 0}$ & $\mathbf{8 0 0}$ & $\mathbf{1 0 0}$ & $\mathbf{1 2 5 0}$ & $\mathbf{1 6 0 0}$ \\
\hline Rock wool & 0.24 & 0.29 & 0.35 & 0.46 & 0.60 & 0.71 & 0.79 & 0.86 & 0.93 \\
Glass wool & 0.25 & 0.25 & 0.25 & 0.30 & 0.35 & 0.42 & 0.50 & 0.65 & 0.77 \\
Raw RTTF & 0.28 & 0.36 & 0.46 & 0.62 & 0.75 & 0.83 & 0.87 & 0.89 & 0.92 \\
Shredded RTTF & 0.28 & 0.33 & 0.40 & 0.57 & 0.72 & 0.82 & 0.89 & 0.93 & 0.95 \\
Purified RTTF & 0.28 & 0.31 & 0.38 & 0.52 & 0.68 & 0.79 & 0.87 & 0.93 & 0.97 \\
\hline
\end{tabular}

Regarding the results, conventional material - RW and GW - performance on sound absorption is lower than RTTF waste. Comparing any fraction of RTTF to GW, results show that alternative materials' performance is better.

\section{CONCLUSION}

To test RTTF performance for sound absorption applications two conventional materials were tested - RW and GW, and one unconventional - RTTF waste. The RTTF was prepared in three options - shredded, raw, and purified RTTF. It was estimated that RW sound absorption at $250 \mathrm{~Hz}$ is 0.24 , while at $1600 \mathrm{~Hz}$ is 0.93 . Secondly, GW sound absorption coefficient at $250 \mathrm{~Hz}$ was 0.25 , and at $1600 \mathrm{~Hz}$ was 0.77 . By comparing those two, RW 
performance on sound absorption is greater than GW.

Sound absorption was measured for raw RTTF, the results were that minimum sound absorption gained at low frequency of $250 \mathrm{~Hz}$, and coefficient was 0.28 , while at $1600 \mathrm{~Hz}$ maximum sound absorption was obtained of 0.92 . Shredded RTTF similar to raw RTTF, sound absorption at $250 \mathrm{~Hz}$ coefficient was 0.28 , the highest 0.95 result obtained at $1600 \mathrm{~Hz}$. Purified RTTF sound absorption coefficient at $250 \mathrm{~Hz}$ frequency was 0.28 , at $1600 \mathrm{~Hz}-0.97$.

By comparing RTTF material options, it was noticed that they perform very similarly. In future study the focus will be put on RTTF purification because sound absorption properties depending on purification level are unknown. Non-acoustic parameters (density, air flow resistivity, porosity) will be investigated in the future studies.

All measured samples were compared, in order to find out which one is the most efficient in sound absorption. RTTF showed results for potential use for sound absorption applications. The results in this study shows that RTTF sound absorption values are similar or better compared to GW and RW.

\section{REFERENCES}

[1] Corredor-Bedoya A. C., Zoppi R. A., Serpa A. L. Composites of scrap tire rubber particles and adhesive mortar-Noise insulation potential. Cement and Concrete Composites 2017:82:45-66. https://doi.org/10.1016/j.cemconcomp.2017.05.007

[2] Frolova O., Salaiová B. Analysis of Road Cover Roughness on "control” Road Section with Crumb Tire Rubber. Procedia Engineering 2017:190:589-596. https://doi.org/10.1016/j.proeng.2017.05.384

[3] Asdrubali F., D’Alessandro F., Schiavoni S. A review of unconventional sustainable building insulation materials. Sustainable Materials and Technologies 2015:4:1-17. https://doi.org/10.1016/j.susmat.2015.05.002

[4] Islam S., Bhat G. Environmentally-friendly thermal and acoustic insulation materials from recycled textiles. Journal of Environmental Management 2019:251:109536. https://doi.org/10.1016/j.jenvman.2019.109536

[5] Vandasova Z., Vencálek O., Puklová V. Specific and combined subjective responses to noise and their association with cardiovascular diseases. Noise \& Health 2016:18:338-346.

[6] Hume K. I., Brink M., Basner M. Effects of environmental noise on sleep. Noise \& Health 2012:14(61):297302. https://doi.org/10.4103/1463-1741.104897

[7] Yankaskas K. Prelude: Noise-induced tinnitus and hearing loss in the military. Hearing Research 2013:295:3-8. https://doi.org/10.1016/j.heares.2012.04.016

[8] Ecopneus. Green Economy Sustainability Report. Rome, 2013.

[9] Landi D., Vitali S., Germani M. Environmental Analysis of Different End of Life Scenarios of Tires Textile Fibers. Procedia CIRP 2016:48:508-513. https://doi.org/10.1016/j.procir.2016.03.141

[10] Shulman V. L. Chapter 21-Tyre Recycling. Waste. Academic Press, 2011:297-320. https://doi.org/10.1016/B978-012-381475-3.10021-X

[11] Dasgupta S., Hammond W. B., Goddard W. A. Crystal structures and properties of nylon polymers from theory. Journal of the American Chemical Society 1996:118(49):12291-12301. https://doi.org/10.1021/ja944125d

[12] Henry B., Laitala K., Klepp I. G. Microfibres from apparel and home textiles: Prospects for including microplastics in environmental sustainability assessment. Science of The Total Environment 2019:652:483-494. https://doi.org/10.1016/j.scitotenv.2018.10.166

[13] Geyer R., Jambeck J. R., Law K.L. Production, use, and fate of all plastics ever made. Science Advances 2017:3(7):e1700782. https://doi.org/10.1126/sciadv.1700782

[14] Taylor M. L., et al. Plastic microfibre ingestion by deep-sea organisms. Scienific Reports 2016:6:33997. https://doi.org/10.1038/srep33997

[15] Bai M. R., Lo Y-Y., Chen Y. S . Impedance measurement techniques for one-port and two-port networks. The Journal of the Acoustical Society of America 2015:138:2279-90. https://doi.org/10.1121/1.4931440

[16] Bujoreanu C., et al. Experimental and theoretical considerations on sound absorption performance of waste materials including the effect of backing plates. Applied Acoustics 2017:119:88-93. https://doi.org/10.1016/j.apacoust.2016.12.010

[17] Amares S., et al. A Review: Characteristics of Noise Absorption Material. Journal of Physics: Conference Series 2017:908:012005. https://doi.org/10.1088/1742-6596/908/1/012005.

[18] Alessandro F. D., Pispola G. Sound absorption properties of sustainable fibrous materials in an enhanced reverberation room. 2005 Congress and Exposition on Noise Control Engineering, Rio de Janeiro, Brazil, 2005. 
[19] Azkorra Z., et al. Evaluation of green walls as a passive acoustic insulation system for buildings. Applied Acoustics 2015:89:46-56. https://doi.org/10.1016/j.apacoust.2014.09.010

[20] Wertel S. J. Experimental Analysis of Noise Reduction Properties of Sound Absorbing Foam. Wisconsin: Universicty of Wisconsin-Stout, 2000.

[21] Mahmoud I. A. Treatment the Effects of Studio Wall Resonance and Coincidence Phenomena for Recording Noisy Speech Via FPGA Digital Filter. Journal of Telecommunications 2010:2:42-8.

[22] Lu T., Xin F. Vibro-Acoustics of Lightweight Sandwich Structures. Springer Tracts in Mechanical Engineering. California: Springer Nature, 2014. https://doi.org/10.1007/978-3-642-55358-5.

[23] Lee Y. E., Joo C. W. Sound Absorption Properties of Thermally Bonded Nonwovens Based on Composing Fibers and Production Parameters. Journal of Applied Polymer Science 2004:92(4):2295-2302. https://doi.org/10.1002/app.20143

[24] Seddeq H. S. Factors Influencing Acoustic Performance of Sound Absorptive Materials. Australian Journal of Basic and Applied Sciences 2009:3:4610.

[25] Soltani P., Zerrebini M. The analysis of acoustical characteristics and sound absorption coefficient of woven fabrics. Textile Research Journal 2012:82:875-882. https://doi.org/10.1177/0040517511402121

[26] Sikora J., Turkiewicz J. Sound absorption coefficients of granular materials. Mechanics and Control 2010:29:149-157.

[27] Malaiškienė J., Nagrockienė D., Skripkiūnas G. Possibilities To Use Textile Cord Waste From Used Tires For Concrete. Journal of Environmental Engineering \& Landscape Management 2015:23(3):183-191. https://doi.org/10.3846/16486897.2015.1057514

[28] Landi D., et al. Reuse scenarios of tires textile fibers: An environmental evaluation. Procedia Manufacturing 2018:21:329-336 https://doi.org/10.1016/j.promfg.2018.02.128.

[29] Marconi M., et al. Reuse of Tires Textile Fibers in Plastic Compounds: Is this Scenario Environmentally Sustainable? Procedia CIRP 2018:69:944-949. https://doi.org/10.1016/j.procir.2017.11.074

[30] Doutres O., et al. Evaluation of the acoustic and non-acoustic properties of sound absorbing materials using a threemicrophone impedance tube. Applied Acoustics 2010:71(6):506-509. https://doi.org/10.1016/j.apacoust.2010.01.007

[31] Umnova O., et al. Deduction of tortuosity and porosity from acoustic reflection and transmission measurements on thick samples of rigid-porous materials. Applied Acousttics 2005:66(6):607-624. https://doi.org/10.1016/j.apacoust.2004.02.005

[32] ISO. Acoustics - Determination of sound absorption coefficient and impedance in impedance tubes - Part 1: Method using standing wave ratio 1996:1-20.

[33] ISO. Acoustics - Determination of sound absorption coefficient and impedance in impedance tubes - Part 2: Transferfunction method. Int Stand 1998:1-28. 